\title{
Validation of a short questionnaire for estimating dietary calcium intakes
}

\author{
H. M. Macdonald • A. J. Black
}

Received: 19 August 2014 / Accepted: 20 August 2014 / Published online: 30 August 2014

(C) International Osteoporosis Foundation and National Osteoporosis Foundation 2014

To the editor

We thank Rasch and co-workers for their supportive comments [1] and the opportunity to clarify our work [2]. We agree with their summary of why the short questionnaire may be useful in clinical practice. Although it is not an accurate estimation of calcium intake, it can distinguish those who have adequate calcium intake. As it tends to underestimate dietary calcium intake compared to the gold standard, further questioning of patients whose diets appear to be lacking by this tool may establish that their dietary calcium intake is adequate. If insufficient, then calcium supplements will be necessary.

With respect to the 250-mg dietary calcium difference between the calcium questionnaire and the gold standard method (food diary), there were 23-26\% whose dietary calcium intakes would have been found to be $250 \mathrm{mg}$ lower by the calcium questionnaire compared to the food diaries, and this number increases to approximately one half when comparing to the food frequency questionnaire (FFQ). Therefore, using the gold standard comparison, we would have saved $75 \%$ of patients from receiving unnecessary calcium supplements (half if comparing against the FFQ). The concerns about cardiovascular risk (although not unequivocal) and other less severe side effects would also affect

H. M. Macdonald

School of Medicine and Dentistry, University of Aberdeen,

Foresterhill, Aberdeen AB25 2ZD, UK

\section{A. J. Black}

NHS Grampian, Aberdeen Royal Infirmary, Ashgrove Road House, Foresterhill, Aberdeen AB25 2ZD, UK

H. M. Macdonald $(\bowtie)$

Musculoskeletal Research, Health Sciences Building, University of Aberdeen, Foresterhill, Aberdeen AB25 2ZD, UK

e-mail: h.macdonald@abdn.ac.uk patient compliance, which is another reason not to supplement those who do not need it. Although further questioning of a patient found to have a low dietary calcium intake by the short questionnaire might help determine whether their calcium is adequate, we recognise that some patients might be still be given calcium supplements they might not necessarily need.

As we indicated in the paper, we would not rely on the higher calcium intake cut-offs $(1,200 \mathrm{mg})$ because there were few subjects in this category (Table 3 shows $12 \%$, which is 14 patients). In the UK, the RNI for calcium is $700 \mathrm{mg}$ a day for adults. This is the amount required to meet $97.5 \%$ of the population's needs. There are no data to support that higher dietary calcium intakes are required as indicated by meta-analyses of studies (although there are issues with regard to standardisation of portion size). Specifically, increments of $300 \mathrm{mg}$ calcium were not associated with fracture risk [3]. Arguments for higher requirements for calcium intakes are primarily based on the fact that studies examining bone treatments included calcium and vitamin D supplementation (although the protocol varies considerably between studies).

Finally, as vitamin D is the nutrient that is usually coprescribed with calcium, and active calcium absorption requires vitamin $\mathrm{D}$, it is important to ensure that patients are vitamin D replete.

\section{References}

1. Letter by Rasch

2. Macdonald HM, Garland A, Burr J, Strachan A, Wood AD, Jamil NA et al (2014) Validation of a short questionnaire for estimating dietary calcium intakes. Osteoporos Int 25(6):1765-73

3. Bischoff-Ferrari HA, Dawson-Hughes B, Baron JA, Burckhardt P, Li $\mathrm{R}$, Spiegelman D et al (2007) Calcium intake and hip fracture risk in men and women: a meta-analysis of prospective cohort studies and randomized controlled trials. Am J Clin Nutr 86(6):1780-90 